\title{
La pedagogía del texto: Enseñanza/aprendizaje del producto final y del proceso de producción verbal
}

\author{
Mercè Pujol-Berché
}

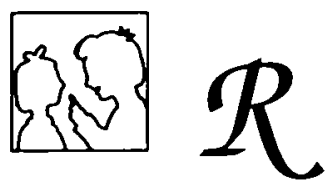

Partiendo de la psicología del lenguaje de inspiración vigotskiana, la autora presenta las principales caracteristicas de la pedagogía del texto. Subraya la necesidad de tener en cuenta en la enseñanza, la diversidad de situaciones de comunicación que produce la diversidad de los discursos existentes, caracterizados tanto por su bomogeneidad como por su beterogeneidad.

De todos es sabido que el lenguaje es la principal herramienta de la educación. Asistimos, desde hace unos años, a un interés cada vez más importante por renovar la enseñanza de la lengua en la escuela. Este interés es debido, en primer lugar, al impulso de las investigaciones sobre la adquisición del lenguaje. En segundo lugar, al incremento de investigaciones, tanto teóricas como empíricas, en lo que se ha designado con el nombre de la pedagogía del texto. Sin embargo, observamos que se ha dejado un poco de lado el estudio de la didáctica de la lengua oral, seguramente por razones sobre todo de índole metodológica: es difícil trabajar con la lengua oral.

La finalidad de mi contribución es doble. En primer lugar, subrayar la necesidad de insertar la didáctica de la lengua (oral y escrita) en una teoría epistemológica sobre el lenguaje. En segundo lugar, presentar, aunque sea suscintamente, las bases sobre las que reposa la pedagogía del texto (oral o escrito), tal y como ésta se está elaborando en el equipo de didáctica de las lenguas de la Universidad de Ginebra. En la primera parte, se presenta, muy brevemente, la teoría sociocognitiva de la adquisición del lenguaje que es la base epistemológica de la psicología del lenguaje de inspiración vygotskiana. En la segunda parte, se subrayan los aspectos más relevantes de la didáctica de la lengua. En la tercera, se define la pedagogía del texto haciendo hincapié tanto en el producto final como en el propio proceso de producción verbal. Finalmente, se esbozan algunas particularidades de la lengua oral en relación a la lengua escrita.

Posición epistemológica: una teoría sociocognitiva de la adquisición del lenguaje

La posición epistemológica que adoptamos es la desarrollada por Vygotski (1934/85', 1935/79), según el cual, el lenguaje se adquiere por y para la comunicación. 
En esta perspectiva, se considera al lenguaje como una herramienta social y se analiza su impacto en el desarrollo individual. Tomar como marco teórico la teoría vigotskiana (Vygotski (1934/85, 1935/79, 1982/91), Alvarez y del Río (1984, 1990), Rivière (1984), Bruner (1985), John-Steiner (1985), Schneuwly y Bronckart (1985), Vila (1987), Wertsch (1988), entre muchos otros) para explicar la aparición, la adquisición y el desarrollo del lenguaje, es reconocer el estatuto fundamentalmente social del funcionamiento humano y el carácter semiótico de la actividad.

En efecto, el estatuto fundamentalmente social del funcionamiento humano tesis de la génesis social del pensamiento - mantiene que el pensamiento y la conciencia son determinados por las actividades realizadas con los demás en un medio social determinado. El carácter semiótico de la actividad (cf. Vila, 1987) - tesis de la mediación - sostiene, en primer lugar, que la mediación hace posible el desarrollo de lo que es natural e inferior a lo que es cultural, social y superior, y, en segundo lugar, que la cooperación social se realiza mediante herramientas semióticas, cuya interiorización construye el pensamiento consciente y regula las otras funciones psíquicas (cf. Schneuwly y Bronckart, 1985).

El funcionamiento social se refiere, por decirlo de una manera muy escueta y simple, al espacio en el cual lo individual y lo social se juntan. También significa que los savoir-faire, los conocimientos del mundo y los conocimientos en general se adquieren gracias a las interacciones con los demás, en las cuales el hablante, sea cual sea su posición, aprende los diferentes modelos comunicativos en vigor en el grupo social de referencia. Este aspecto tiene, efectivamente, una función determinante en el desarrollo del lenguaje, ya que éste no puede aparecer sin que los niños participen en interacciones (cf. Pujol-Berché, 1993). El niño adquiere su(s) lengua(s) en el marco de una actividad social, gracias a las interacciones que éste tiene con otras personas de su mismo grupo social y que es propicia a lo que Bruner (1983) llama formatos, siendo el lenguaje un tipo de actividad. Desde esta perspectiva, la adquisición del lenguaje, tanto oral como escrito, es un proceso dinámico. La adquisición de la lengua oral y de la lengua escrita forman parte de una misma clase de desarrollo verbal y se encuentran internamente unidos (cf. Schneuwly, 1992).

A partir de este posicionamiento epistemológico, la psicología del lenguaje de inspiración vygotskiana tiene como finalidad la explicación de los observables, es decir «de los comportamientos de lenguaje $e^{2}$ materialmente producidos por sujetos hablantes, en el marco de las restricciones impuestas por una lengua natural» (Bronc-

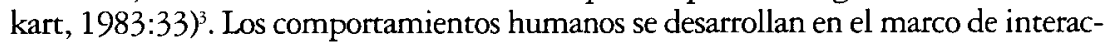
ciones diversas o actividades, que pueden definirse como un conjunto organizado de comportamientos. La actividad verbal es una de dichas actividades con finalidad comunicativa. Como lo recuerda Bronckart, en la perspectiva de Leontiev (1979), «la noción de actividad ${ }^{4}$ hace referencia a las formas más generales de organización funcional de los comportamientos a través de los cuales, los miembros de una especie tienen acceso al mundo objetivo y construyen una representación interna (o conocimiento)» (1992:31). La actividad se desarrolla bajo la forma de acciones finalizadas, de intenciones y según las reglas de un grupo social determinado. Las acciones son modalidades sociales prácticas, las «acciones sociales con sentido» constituyen una intervención en un contexto social dado. Así pues, los sistemas de actividad están definidos por la cultura y por las normas existentes en la misma. En efecto, según Bajtín (1977), cualquier sistema de normas exite únicamente en relación a la conciencia subjetiva de los individuos que pertenecen a una colectividad regida por normass.

\section{Los fundamentos de la didáctica de la lengua}

Una vez precisado, a nivel psicológico, el marco teórico que sirve a la psicología del lenguaje para elaborar propuestas didácticas en lengua, es necesario precisar aquellos modelos o teorías lingüísticas que servirán de base a tal elaboración. Debe- 
mos aclarar ante todo, y como ya ha sido puesto en evidencia con anterioridad, que la didáctica es una disciplina autónoma (cf. Bronckart, 1989) y que no debe procederse a una aplicación directa de las últimas investigaciones en lingüística o en psicología a la didáctica, ya que ello no siempre han dado buenos frutos (cf. Pujol-Berché, 1992b). A pesar de ello, y huyendo de cualquier tipo de aplicación, la didáctica de la lengua debe darse como referencia modelos lingüísticos a partir de los cuales, y previa selección, elaborar propuestas concretas. En el estado actual de conocimientos, la lingüística textual, centrada en el texto (y no en la frase) y en la configuración de unidades lingüísticas que los componen proporciona un buena herramienta a la didáctica, al tomar en consideración las relaciones entre las diferentes unidades lingüísticas - el co-texto (lingüístico) - y dichas unidades y los parámetros extralingüústicos - el contexto (extralingüístico).

En resumen, la didáctica de la lengua que se propone desde esta perspectiva, parte, en primer lugar, de la psicología del lenguaje de inspiración vygotskiana interaccionismo social - y, en segundo lugar, de la lingüística textual - o para ser más precisas del funcionamiento de los discursos (cf. Bronckart y cols, 1985). La lingüística textual ha puesto en evidencia que a partir de un número finito de unidades (la lengua considerada como sistema o como código) se produce una gran diversidad de discursos diferentes (de hecho, infinitos). En efecto, como lo subraya Bronckart (1991), la diversidad textual no es otra cosa que un reflejo de la diversidad de acciones humanas, y una teoría de los discursos (sobre la cual reposa, en parte, la pedagogía del texto) debe articularse a una teoría sobre las acciones comunicativas.

Dicha articulación, que ha sido conceptualizada por Bronckart y cols. (op.cit.), es resumida brevemente a continuación. La hipótesis general subyacente es que la variedad de las acciones verbales depende fundamentalmente de la diversidad de contextos; ello no debe comprenderse, en modo alguno, como un reduccionismo de un efecto mecánico de los parámetros del mismo. La acción verbal es el resultado de un cierto número de decisiones discursivas. La actividad verbal, que se inicia en una situación de comunicación a partir de la cual el proceso de comunicación/representación tiene lugar, se articula con el extralenguaje, concepto teórico que se define como todas aquellas entidades mundanas externas a la lengua. El tratamiento de los parámetros extralingüísticos se traduce en una serie de unidades lingüísticas que se organizan en un texto. Para evitar confusiones a este nivel, nos parece necesario distinguir entre discurso y texto. Según Bronckart y cols. (op.cit.), éste último es un objeto formal sobre el que trabajamos (psicólogos, lingüistas, pedagogos de la lengua, etc.), mientras que el discurso es «un espacio intermedio entre la infinita diversidad de los objetos y de los hechos (...) y la infinita diversidad de las prácticas y de las situaciones» (Bronckart, 1987:37)6.

A nivel psicológico, una acción verbal tiene un objetivo. Debido al hecho de que exite una infinidad de objetivos comunicativos, existe, también, una infinidad de acciones verbales. Cada acción verbal se produce en un espacio comunicativo con los parámetros de la situación material de enunciación y de la interacción (sobre todo el rol de los interlocutores). Como una accción verbal se materializa en una lengua determinada, el hablante deberá elegir, por una parte, un cierto tipo de discurso según el objetivo propuesto (eficacia) y la representación del espacio comunicativo (pertinencia), y, por otra parte, las diferentes unidades lingüísticas que le ofrece su lengua.

Como acabamos de mencionar, la diversidad de los discursos se explica por la infinidad de acciones verbales existentes. A pesar de poder distinguir tipos discursivos que se contrastan (discurso teórico, discurso en situación, narración, etc.), es importante subrayar que, incluso dentro de un mismo grupo de discursos - arquetipos (definido por las deciciones que el hablante ha llevado a cabo teniendo en cuenta la finalidad comunicativa, la representación de los parámetros extralingüísticos y la 
representación del objeto de discurso), pueden existir algunas características lingüísticas diferentes. Es decir, que no sólo existe diversidad entre los discursos, sino que existe también diversidad intra-tipo discursivo. Cada texto presenta sus propias características que comparte con otros textos dentro de la misma categoría de discurso - bomogeneidad -, y otras características que lo hacen distinto - beterogeneidad. Algunos textos son homogénos pero la mayoría son heterogéneos: presentan secuencias cuyas características discursivas son distintas. En resumen, no sólo existe diversidad textual, sino también heterogeneidad.

Partiendo de esta constatación, una buena didáctica de la lengua (oral y escrita) ha de tener en cuenta ambos aspectos: diversidad y heterogeneidad. El problema que se plantea entonces es elegir entre la gran diversidad de discursos, aquellos que deben ser motivo de aprendizaje. Sería ilusorio e imposible trabajarlos todos (véase Besson y Canelas-Trevisi, en este mismo número). La didáctica tiene que elegir aquellos que serán motivo de enseñanza. Los criterios de elección son numerosos. Nos podríamos inclinar por los más representativos numéricamente o por aquellos que posiblemente serán más utilizados en la vida social adulta. Ambos criterios podrían ser válidos. Lo más importante es, en primer lugar, definir los objetivos que queremos conseguir y que queremos que los alumnos alcancen; luego, en función de dichos objetivos, elegir aquellos discursos representativos de la totalidad de discursos propios del grupo social. Ante esto se impone pues, un criterio de elección en función de una serie de aspectos estrechamente vinculados a los objetivos de la enseñanza y a la eficacia de la misma.

\section{La pedagogía del texto orientada tanto hacia el producto final como hacia el proceso de producción}

Los elementos que acabamos de definir y los distintos aspectos que conforman la línea de partida han permitido definir y elaborar la pedagogía del texto. En efecto, la propuesta pedagógica elaborada en la Unidad de didáctica de las lenguas de la Universidad de Ginebra (cf. Commission Pédagogie du texte (1985, 1988); Bronckart (1989, 1991); Bronckart y Schneuwly (1991), entre otras publicaciones) insiste tanto en los arquetipos discursivos (discurso en situación, discurso teórico, etc.) según la operación de anclaje efectuada (la implicación directa en la interacción social produce el discurso en situación, mientras que la autonomía en relación a la interacción social, produce el discurso teórico), como en la heterogeneidad (no todos los discursos en situación presentan exactamente las mismas características lingüísticas).

La pedagogía del texto surge de la necesidad de adaptar los aprendizajes escolares en materia de lengua a la diversidad de situaciones de comunicación que los alumnos deberán afrontar en su vida cotidiana. La finalidad de la misma es proporcionar a los alumnos los diferentes tipos de discurso (tipología textual) que necesitarán dominar para enfrentarse a su vida social, y más tarde profesional. Abordar la enseñanza de la expresión supone entrar en materia sobre la diversidad discursiva y su carácter multidimensional.

Como ya hemos dicho en otra ocasión (Dolz y Pujol-Berché, 1991), la enseñanza/aprendizaje de la lengua se inscribe en un proyecto pedagógico global, con una serie de objetivos de aprendizaje y una secuencia didáctica compuesta por diferentes módulos cada uno de ellos con una finalidad y un objetivo específicos y bien definidos. Cada módulo contiene ejercicios relativos a cada una de las dimensiones discursivas en las que el trabajo global sobre los textos se alterna con el trabajo local sobre las unidades lingüísticas. En resumen, el objetivo de la pedagogía del texto es esta- 
blecer secuencias didácticas destinadas a mejorar las producciones orales y escritas de los alumnos.

De manera muy breve, enunciaremos algunas de las ventajas de la pedagogía del texto. En primer lugar, propone una definición clara y precisa del producto final esperado (en función del tema a tratar, la finalidad perseguida por la acción discursiva, el destinatario, etc.) y una gama de medios didácticos para alcanzarlo. Las tareas solicitadas se adaptan, en la medida de lo posible, a situaciones auténticas de comunicación. La pedagogía del texto presenta como prioritaria, la articulación de las actividades de lectura y producción de textos (orales y escritos) a las distintas disciplinas escolares, y concierne no sólo el área de lengua sino la totalidad del currículum (cf. véase Vila y Vilà, en este mismo número).

Para que los alumnos puedan elaborar un texto según las expectativas que los profesores tienen (y que corresponderá a los modelos en vigor en el grupo social de pertenencia), es necesario, en primer lugar, darles una consigna lo más precisa posible (cf. Besson y Canelas-Trevisi, en este número), con lo cual estamos trabajando hacia el producto que deseamos obtener. Para ello es necesario, precisar, por una parte, la finalidad de la acción verbal (informar, explicar, divertir, etc.), el (los) destinatario(s) de la misma (y por lo tanto tendremos en cuenta sus roles sociales) y el(los) enunciador(es) (habla el mismo alumno o habla en boca de otro - situación típica de los juegos de roles -) y, por otra parte, el espacio-tiempo en donde se llevará a cabo dicha acción verbal. Para decirlo de otra forma, nos estamos situando en un primer nivel de todas aquellas operaciones que tendrá que llevar a cabo el alumno durante la producción de su texto: procesos cognitivos de representación con las operaciones de contextualización (espacio-tiempo, finalidad, destinatarios, etc.) y las operaciones de referenciación (tema del discurso). Este primer nivel de operaciones permitirá llegar a un segundo nivel y crear una base de orientación en la cual el alumno gestionará globalmente su texto. Se llevarán a cabo una serie de operaciones de anclaje y de planificación cognitiva y comunicativa (conocimientos sobre los modelos y prácticas del grupo social). Si él es el único hablante, tendrá que monogestionar su discurso; si hay varios interlocutores, éstos lo tendrán que construir juntos y cada uno de ellos tendrá en cuenta las palabras del otro dando lugar a un discurso poligestionado. El primero es característico del lenguaje escrito (aunque también existe en el lenguaje oral: pensemos en un conferenciante, en un orador), mientras que el segundo es más característico del lenguaje hablado y más concretamente de los discursos en situación, de los intercambios, etc. en los que los participantes presentes en la interacción construyen conjuntamente su texto). Finalmente, en el tercer nivel de operaciones - operaciones de linearización -, gestión local del texto, el hablante elige aquellas unidades lingüísticas que le permiten textualizar su texto (cohesión, conexión y modalización), este nivel se refiere más específicamente a los conocimientos que se poseen de la lengua.

Al tener en cuenta este modelo de funcionamiento de los discursos y sus tres niveles de operaciones estamos no sólo trabajando el producto final, sino también, y lo que me parece muy importante, el proceso mismo de la producción verbal. En efecto, si la consigna dada al inicio de la actividad sirve para definir exactament el producto final deseado (o para decirlo de otra manera y utilizando la célebre frase de Fishman (1967) para precisar: ¿quién habla a quién, de qué, (cómo), cuándo y dónde?), la toma de conciencia de los tres niveles de operaciones sirven para que el alumno pueda trabajar el proceso mismo de producción (tanto oral como escrita). Los diferentes módulos propuestos por la pedagogía del texto permiten que el alumno tome conciencia, en primer lugar, del funcionamiento de los discursos (modelos) y en segundo lugar, de su propia actividad (su producción-proceso según los modelos en vigor). 


\section{Algunas particularidades de la didáctica de la lengua oral}

Si bien lo dicho anteriormente es válido tanto para la lengua oral como para la lengua escrita, nos parece que una enseñanza orientada hacia la lengua oral tiene que tener en cuenta, además y sin ánimo de exhaustividad, los aspectos siguientes?.

En primer lugar, se hará hincapié en el hecho que la lengua oral y la lengua escrita pertenecen a una misma lengua. Ninguna de ellas es mejor, cada una es utilizada según las necesidades de la situación de comunicación y el canal elegido para la transmisión del mensaje. Ambas presentan características en común, pero también algunas características diferentes. Así pues, ciertas unidades linguiísticas son más frecuentes en la lengua oral que en la lengua escrita. Además, la sintaxis de la lengua oral está formada, entre otras particularidades, por repeticiones, dudas, dislocaciones, etc.

En segundo lugar, los alumnos deben comprender que lo que aprenden en clase de lengua no es inútil, sino que por el contrario, lo pueden (y deben) utilizar en cualquier otra asignatura (cf. Pujol-Berché, 1992a). Esto significa que debemos huir de convertir la clase de lengua en una reflexión metalingüística sobre la lengua o sobre el funcionamiento de la misma.

En tercer lugar, y como lo demuestran todos los artículos de este monográfico (véase también Wirthner, Martin y Perrenoud, 1991), la enseñanza de la lengua oral debe llevarse a cabo de manera sistemárica. «Hablar por hablar» no conduce a nada. Hay que dar la palabra a los alumnos pero además les debemos proporcionar las herramientas necesarias para que su toma de palabra sea pertinente y adecuada a la finalidad comunicativa que se quiere conseguir (explicar a un compañero cómo funciona la máquina expendedora de coca-colas, argumentar delante de sus padres que, a cierta edad, es necesario contar con un poco de dinero semanal para los propios gastos, etc.).

En cuarto lugar, es necesario que la didáctica de la lengua oral se complemente con la de la lengua escrita. Muchas actividades cotidianas necesitan simultánemante ambas: tomar apuntes mientras habla el profesor, resumir oralmente el artículo del periódico que acabamos de leer, etc.

En quinto y último lugar, en los discursos orales poligestionados, los alumnos han de tomar conciencia de los roles sociales que se les atribuye y de aquellos que son atribuidos a sus interlocutores: modalizaciones en el discurso (no nos dirigimos de la misma forma a un compañero de clase o al director de la escuela, y por lo tanto, no utilizamos exactamente las mismas unidades lingüísticas).

\section{A modo de conclusión}

Hemos querido presentar de manera muy sucinta, las bases epistemológicas sobre las que se apoya la pedagogía del texto. Al hacerlo, hemos querido tener en cuenta tanto el lenguaje oral como el lenguaje escrito porque creemos que la enseñanza de ambos debe llevarse a cabo de forma sistemática, con objetivos precisos. En efecto, la vida cotidiana nos muestra que no hay una separación neta entre ambos y que en muchas ocasiones sendos son utilizados casi de forma simultánea. Al tomar la pedagogía del texto como medio o técnica para la enseñanza, hemos querido huir de convertir la clase de lengua en una pura reflexión metalingüística; antes bien, la pedagogía del texto quiere adaptar la enseñanza de la misma a las necesidades de los alumnos. 


\section{Notas}

1. La primera fecha se refiere a la publicación original rusa, la segunda a la traducción y a la edición utilizada.

2. En cursiva en el texto.

3. Nuestra traducción.

4. En cursiva en el texto.

5. Véase François (1989) y Wertsch (1991) para una exposición detallada de la contribución de Bakhtine más allá de Vigotski.

6. La traducción es nuestra.

7. El lector interesado por la psicopedagogía de la lengua oral, consultará, entre otros, Tough (1987); Wirthner, Martin y Perrenoud (1991); del Río (1993).

\section{Referencias}

Alvarez, A. y del Rio, P. (Coord.) (1984) Vygotski: cincuenta años después, Infancia y Aprendizaje, $\mathrm{n}^{\circ}$ especial, 27-28

Alvarez, A. y del RIO, P. (1990) «Actividad y lenguaje. El diseño cultural como marco para un programa de enseñanza del inglés como lengua extranjera», Comunicación, lenguaje y educación, 7-8, pp. 99125

Bajtin, M. (1977) Le marxisme et la pbilosopbie du langage, París, Minuit

BroncKarT, J.-P. (1983) «Cas et prédicats. Une étude de psychologie du langage», en La Linguistique, 19, pp. 29-53

BRONCKART, J.-P. (1987) «Interactions, discours, significations», Langue française, 74, pp. 29-50

BRONCKART, J.-P. (1989) «Du statut des didactiques des matières scolaires», Langue française, 82, pp. 53-66

BronCKarT, J.-P. (1991) «Perspectives et limites d'une diversification de l'enseignement du français», Études de Linguistique Appliquée, 83, pp. 63-74

BRoNCKART, J.-P. (1992) «El discurso como acción. Por un nuevo paradigma psicolingüístico», Anuario de Psicología, 3, pp. 3-48

BRONCKART, J.-P. y SCHNEUWLY, B. (1991) «La didactique du français langue maternelle; l'émergence d'une utopie indispensable», Education et recherche, 13, pp. 8-26

Bronckart, J.-P.; Bain, D.; SCHNeUwly, B.; Davaud, C. y PasQuier, A. (1985) Le fonctionnement des discours, Paris-Neuchâtel, Delachaux y Niestlé

BRUNER, J.S. (1983) Le développement de l'enfant: savoir faire, savoir dire, París, Presses Universitaires de France

BRUNER, J.S. (1985) «Vygotsky: a historical and conceptual perspective», en WERTSCH, J.V. (Ed.) Culture, communication, and cognition: Vygotskyan perspectives, Cambridge, Cambridge University Press, pp. $21-34$

Commission Pédagogie Du TeXte (1985) Contributions à la pédagogie du texte I, Ginebra, Cahiers de la Section des Sciences de l'Éducation, $n^{\circ} 40$

COMmission PÉDAGogie Du TeXte (1988) Contributions à la pédagogie du texte II, Ginebra, Cahiers de la Section des Sciences de l'Éducation, $n^{\circ} 52$

Dolz, J. y Pujol-BerCHé, M. (1991) «La enseñanza de la lengua de origen: propuestas de renovación didáctica», en SIGUAN, M. (Coord.) La enseñanza de la lengua, Barcelona, ICE/Horsori, pp. 91-97

FISHMAN, J.A. (1967) «Who speaks what language to whom and when», La Linguistique, 2, pp. 67-88

FrançoIs, F. (1991) «Le dialogue en maternelle. Mise en mots et enchaînements», en WIRTHNER, M.; Martin, D. y Perrenoud, Ph. (Eds) (1991) Parole étouffée, parole libérée. Fondements et limites d'une pédagogie de l'oral, París/Neuchâtel, Delachaux y Niestlé, pp. 55-80

JOHN-STEINER, V. (1985) «The road to competence in an alien land: a Vygotskyan perspective on bilingualism", en WERTSCH, J.V. (Ed.) Culture, communication, and cognition: Vygotskyan perspectives, Cambridge, Cambrige Univesity Press, pp. 348-371

Leontiev, A.N. (1979) "The Problem of Activity in Psychology», en WERTSCH, J.M. (Eds) The Concept of Activity in Soviet Psychology, New York, Sharpe, pp. 37-71

Pujol-Berché, M. (1992a) «Algunas reflexiones sobre la didáctica de la lengua oral», Comunicación, lenguaje y educación, 16, pp. 119-126

PujOL-BERCHE, M. (1992b) «Quelques réflexions à propos des rapports entre la didactique de la langue maternelle et la didactique de la langue seconde et/ou étrangère», Education et recherche, 1, pp. 81-92

Pujol-Berché, M. (1993) «Interaction bilingue et acquisition simultanée de deux langues", AILE, 2, pp. 109-142

Rto del, M.J. (1993) Psicopedagogia de la lengua oral: Un enfoque comunicativo, Barcelona, ICE/Horsori

Riviere, A. (1984) La psicologia de Vygotski, Madrid, Aprendizaje/Visor 
SCHNEUWLY, B. (1992) «La concepción vigotskiana del lenguaje escrito", Comunicación, lenguaje y educación, 16, pp. 49-60

SCHNEUWLY, B. y BRONCKART, J.-P. (Eds) (1985) Vygotsky aujourd'bui, París/Neuchâtel, Delachaux y Niestlé

TOUGH, J. (1987) El lenguaje oral en la escuela. Una guia de observación y actuación para el maestro, Madrid, Aprendizaje/Visor

VILA, I. (1987) La funció semiòtica de la ment, Vic, Eumo

VyGotsky, L.S. (1934/1985) Pensée et langage, París, Messidor, Editions Sociales

VYGOTSKJ, L.S. (1935/79) El desarrollo de los procesos psicológicos superiores, Barcelona, Crítica

VYGoTSKI, L.S. (1982/91) Obras escogidas, Vol. I Problemas teóricos y metodológicos de la psicología, Madrid, Aprendizaje/Visor y Ministerio de Educación y Ciencia

WERTSCH, J.V. (1988)Vygotsky y la formación social de la mente, Barcelona, Paidós

WERTSCH, J.V. (1991) Voices of the mind: A sociocultural approach to mediated action, Londres, Hervester Wheatsheaf

Wirthner, M.; Martin, D. y Perrenoud, Ph. (Eds) (1991) Parole étouffée, parole libérée. Fondements et limites d'une pédagogie de l'oral, París/Neuchâtel, Delachaux y Niestlé.

\section{La pedagogía del texto: Enseñanza/Aprendizaje del producto final y del proceso de producción verbal Mercè Pujol-Berche CL\&E, 1994, 23, pp. 9-16}

Resumen: Este artículo presenta las principales características de la pedagogía del texto. Ésta tiene en cuenta tanto la diversidad de los discursos, como la heterogeneidad de los mismos. El objetivo de la pedagogía del texto, que se inscribe en un proyecto pedagógico global, es establecer secuencias didácticas destinadas a mejorar las producciones orales y escritas de los alumnos.

Datos sobre el autor: Mercè Pujol ha trabajado durante muchos años en la Unidad de Didáctica de las lenguas de la Universidad de Ginebra. Sus ámbitos de investigación son la adquisición del lenguaje (adquisición simultánea de dos primeras lenguas y adquisición de lenguas extranjeras), la didáctica de lenguas extranjeras y la sociolingüística.

Dirección: Facultad de Psicología y Pedagogía, Universidad Ramon Llull, c/ Cister 24-34, 08025 Barcelona.

(C) PERMISOS PARA CITAR O REPRODUCIR EN OTRAS FUENTES: Se pueden citar libremente hasta 500 palabras. Para reproducir una porción de texto mayor, figuras o ilustraciones, se deberá pedir permiso por escrito a la revista, especificando el uso al que se destina el texto. En todos los casos, se deberá citar el copyright de $C L \& E$. En el caso de artículos o textos que hayan sido a su vez reproducidos en $C L \& E$ los interesados deberán dirigirse tanto a los detentadores del copyright original como a $C L \& E$, en el caso de que se quiera hacer uso de la traducción. FOTOCOPIAS: Para todo lo relacionado con el uso mediante fotocopia del material de esta revista, deberán dirigirse a: CEDRO, C/ José Marañón, 10, $3 .^{\circ}$ Izda. Tel. 59415 75. Fax 4453567 